\title{
Use of Smartphones and Mobile Devices in Hospitalized Patients: Untapped Opportunities for Inpatient Engagement
}

\author{
Steven Ludwin, MD*, S. Ryan Greysen, MD, MHS, MA
}

Division of Hospital Medicine, University of California, San Francisco, San Francisco, California.

Over $90 \%$ of Americans own mobile phones, and their use for internet access is rising rapidly $31 \%$ in $2009,63 \%$ in 2013). ${ }^{1}$ This has prompted growth in mobile health (mHealth) programs for outpatient settings, ${ }^{2}$ and similar growth is anticipated for inpatient settings. ${ }^{3}$ Hospitals and the healthcare systems they operate within are increasingly tied to patient experience scores (eg, Hospital Consumer Assessment of Healthcare Providers and Systems, Press Ganey) for both reputation and reimbursement. ${ }^{4,5}$ As a result, hospitals will need to invest future resources in a consumer-facing digital experience. Despite these trends, basic information on mobile device ownership and usage by hospitalized patients is limited. This knowledge is needed to guide successful mHealth approaches to engage patients in acute care settings.

\section{METHODS}

We administered a 27-question survey about mobile device use to all adult inpatients at a large urban California teaching hospital over 2 dates (October 27, 2013 and November 11, 2013) to create a crosssectional view of mobile device use at a hospital that offers free wireless Internet (WiFi) and personal health records (Internet-accessible individualized medical records). Average census was 447 , and we excluded patients for: age under 18 years (98), admission for neurological problems (75), altered mental status (35), non-English speaking (30), or "unavailable" if patients were not in their room after 2 attempts spaced 30 to 60 minutes apart (36), leaving 173 eligible. We performed descriptive statistics and unadjusted associations $\left(\chi^{2}\right.$ test) to explore patterns of mobile device use.

\section{RESULTS}

We enrolled 152 patients (88\% response rate): 77 $(51 \%)$ male, average age 53 years (19-92 years), 84 $(56 \%)$ white, $115(75 \%)$ with Medicare or commer-

\footnotetext{
*Address for correspondence and reprint requests: Steven Ludwin, MD, Division of Hospital Medicine, University of California, San Francisco, 533 Parnassus Avenue, Box 0131, San Francisco, CA 94113; Telephone: 415-476-4814; Fax: 415-502-1963; E-mail: steven.ludwin@ucsf.edu

Additional Supporting Information may be found in the online version of this article.

Received: November 25, 2014; Revised: March 18, 2015; Accepted: March 24, 2015

2015 Society of Hospital Medicine DOI 10.1002/jhm.2365

Published online in Wiley Online Library (Wileyonlinelibrary.com).
}

cial insurance. We found $85(56 \%)$ patients brought a smartphone, and $82 / 85(95 \%)$ used it during their hospital stay. Additionally, $41(27 \%)$ patients brought a tablet, and 29 (19\%) brought a laptop; usage was $37 / 41(90 \%)$ for tablets and 24/29 (83\%) for laptops. One hundred three $(68 \%)$ patients brought at least 1 mobile computing device (smartphone, tablet, laptop) during their hospital stay. Overall device usage was highest among oncology patients $(85 \%)$ and lowest among medicine patients (54\%) (Table 1). Device usage also varied by age ( $<65$ years old: $79 \%$ vs $\geq 65$ years old: $27 \%$ ), insurance status (private/Medicare: $70 \%$ vs Medicaid/other: 59\%), and race/ethnicity (white: $73 \%$ vs non-white: $62 \%$ ), although only age was statistically significant $(P<0.01 ;$ all others $>0.05$ ).

Of the patients with mobile devices (smartphone, tablet, laptop), 97/103 (94\%) used them during their hospitalization and for a wide array of activities (Figure 1): $47 / 97$ (48\%) accessed their personal health record (PHR), and most of these patients (38/47, $81 \%$ ) reported this improved their inpatient experience. Additionally, $43 / 97$ (44\%) patients used their mobile devices to search for information about doctors, conditions, or treatments; most of these patients $(39 / 43,91 \%)$ used Google to search for this information, and most $29 / 43(67 \%)$ felt this information made them more confident in their care.

\section{COMMENT}

Over two-thirds of patients in our study brought and used 1 or more mobile devices to the hospital. Despite this level of engagement with mobile devices, relatively few inpatients used their device to access their online PHR, which suggests information technology access is not the leading barrier to PHR access or mHealth engagement during hospitalization. In light of growing patient enthusiasm for PHRs, ${ }^{6,7}$ this represents an untapped opportunity to deliver personalized, patient-centered care at the hospital bedside.

We also found that among the patients who did access their PHR on their mobile device, the vast majority $(38 / 47,81 \%)$ felt it improved their inpatient experience. Our PHR provides information such as test results and medications, but our survey suggests a number of patients look for health information, such as patient education tools, medication references, and provider information, outside of the PHR. For those patients, $29 / 43(67 \%)$ felt these health-related 
TABLE 1. Device Ownership and Use Overall Among the Three Largest Hospital Services

\begin{tabular}{|c|c|c|c|c|c|}
\hline & Total, $\mathrm{N}=152$ & Medicine, $n=39$ & Surgery, $n=47$ & Oncology, $n=34$ & All Others, $n=32^{*}$ \\
\hline \multicolumn{6}{|l|}{ Demographics } \\
\hline Average age, y (range) & $53.2(19-92)$ & $55.7(20-92)$ & $51.7(19-79)$ & $51.2(23-77)$ & $53.9(25-84)$ \\
\hline Medicare or commercial insurance & $75 \%(115)$ & $64 \%(25)$ & $87 \%(41)$ & $76 \%(26)$ & $72 \%(23)$ \\
\hline Medicaid, other, or no insurance & $25 \%(37)$ & $36 \%(14)$ & $13 \%(6)$ & $24 \%(8)$ & $28 \%(9)$ \\
\hline Non-white race/ethnicity & $44 \%(68)$ & $56 \%(22)$ & $36 \%(17)$ & $38 \%(13)$ & $50 \%(16)$ \\
\hline Female gender & $49 \%(75)$ & $49 \%(19)$ & $45 \%(21)$ & $47 \%(16)$ & $59 \%(19)$ \\
\hline \multicolumn{6}{|l|}{ Device ownership/usage } \\
\hline Own smartphone & $62 \%(94)$ & $54 \%(21)$ & $66 \%(31)$ & $74 \%(25)$ & $53 \%(17)$ \\
\hline Brought smartphone & $55 \%(83)$ & $41 \%(16)$ & $60 \%(28)$ & $71 \%(24)$ & $48 \%(15)$ \\
\hline Brought laptop & $19 \%(29)$ & $18 \%(7)$ & $11 \%(5)$ & $41 \%(14)$ & $10 \%(3)$ \\
\hline Brought tablet & $27 \%(41)$ & $18 \%(7)$ & $26 \%(12)$ & $50 \%(17)$ & $16 \%(5)$ \\
\hline Brought $\geq 1$ above devices & $68 \%(103)$ & $54 \%(21)$ & $68 \%(32)$ & $85 \%(29)$ & $68 \%(21)$ \\
\hline Ever used an "app" & $63 \%(95)$ & $51 \%(20)$ & $72 \%(34)$ & $79 \%(27)$ & $45 \%(14)$ \\
\hline Ever used an "app" for health purposes & $22 \%(34)$ & $18 \%(7)$ & $21 \%(10)$ & $24 \%(8)$ & $29 \%(9)$ \\
\hline Accessed PHR with mobile device & $31 \%(47)$ & $26 \%(10)$ & $26 \%(12)$ & $47 \%(16)$ & $29 \%(9)$ \\
\hline
\end{tabular}

NOTE: Abbreviations: PHR, personal health record. *Other services surveyed: cardiology, obstetrics and gynecology, and hepatology.

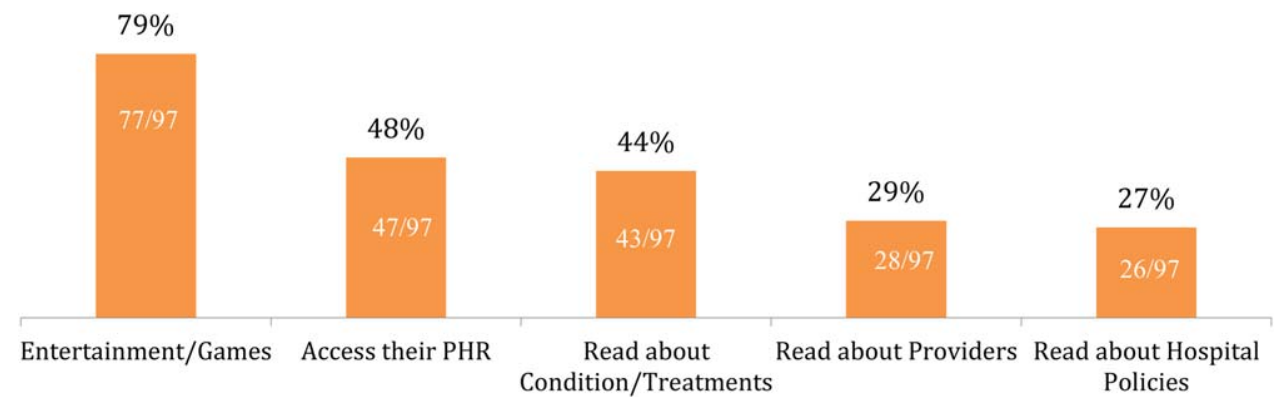

FIG. 1. What do hospitalized patients do with their mobile devices $(n=97)$ ? Abbreviations: PHR, personal health record.

searches improved their experience. Although we did not ask patients why they used Web searches outside their PHR, we believe this suggests that patients desire more information than currently available via the PHR. Although this information might be difficult to incorporate into the PHR, at minimum, hospitals could develop mobile applications to provide patients with basic information about their providers and conditions. Beyond this, hospitals could develop or adopt mobile applications that align with strategic priorities such as improved physician-provider communication, reduced hospital readmissions, and improved accuracy of medication reconciliation.

Our study has limitations. First, although we used a cross-sectional, point-in-time approach to canvas the entire adult population in our hospital on 2 separate dates, our study was limited to 1 large urban hospital in California; device ownership and usage may vary in other settings. Second, although our hospital provides free WiFi, we did not assess whether patients experienced any connectivity issues that influenced their device usage patterns. Finally, we did not explore questions of access, ownership, and usage of mobile computing devices for family and friends who visited inpatients in our study. These questions are ripe for future research in this emerging area of mHeath.

In summary, our study suggests a role for hospitals to provide universal WiFi access to patients, and a role for both hospitals and healthcare providers to promote digital health programs. Our findings on mobile device use in the hospital are consistent with the growing popularity of mobile device usage nationwide. Patients are increasingly "wired" for new opportunities to both engage in their care and optimize their hospital experience through use of their mobile computing devices. Hospitals and providers should explore this potential for engagement, but may need to explore local trends in usage to target specific service lines and patient populations given differences in access and use.

\section{Acknowledgements}

The authors acknowledge contributions by Christina Quist, MD, and Emily Gottenborg, MD, who assisted in data collection.

Disclosures: Data from this project were presented at the 2014 Annual Scientific Meeting of the Society of Hospital Medicine, March 25, 2014 in Las Vegas, Nevada. The authors have no conflicts of interest to declare relative to this study. Dr. Ludwin, MD had full access to all data in the study and takes responsibility for the integrity of the data and the accuracy of the data analysis. This project by Drs. Ludwin and 
Greysen was supported by grants from the University of California, San Francisco (UCSF) Partners in Care (Ronald Rankin Award) and the UCSF Mount Zion Health Fund. Dr. Greysen is also funded by a Pilot Award for Junior Investigators in Digital Health from the UCSF Dean's Office, Research Evaluation and Allocation Committee (REAC). Additionally, Dr. Greysen receives career development support from the National Institutes of Health (NIH)-National Institute of Aging (NIA) through the Claude D. Pepper Older Americans Independence Center at UCSF Division of Geriatric Medicine (\#P30AG021342 NIH/NIA), a Career Development Award (1K23AG045338-01), and the NIH-NIA Loan Repayment Program.

\section{References}

1. Device ownership over time. Pew Research Center. Available at: http://www.pewinternet.org/data-trend/mobile/device-ownership. Accessed April 3, 2014.
2. Free C, Phillips G, Watson L, et al. The effectiveness of mobile-health technologies to improve health care service delivery processes: a systematic review and meta-analysis. PLoS Med. 2013;10(1):e1001363.

3. Steinhubl SR, Muse ED, Topol EJ. Can mobile health technologies transform health care? JAMA. 2013;310(22):2395-2396.

4. Look ahead to succeed under VBP. Hosp Case Manag. 2014;22(7): 92-93.

5. Bardach NS, Asteria-Penaloza R, Boscardin WJ, Dudley RA. The relationship between commercial website ratings and traditional hospital performance measures in the USA. BMJ Qual Saf. 2013;22(3):194202.

6. Zarcadoolas C, Vaughon WL, Czaja SJ, Levy J, Rockoff ML. Consumers' perceptions of patient-accessible electronic medical records. J Med Internet Res. 2013;15(8):e168.

7. Schickedanz A, Huang D, Lopez A, et al. Access, interest, and attitudes toward electronic communication for health care among patients in the medical safety net. J Gen Intern Med. 2013;28(7):914-920. 